\title{
Sincronía y diacronía de los sonidos retroflejos del quechua huanca desde la fonética articulatoria
}

\section{Synchrony and diachrony of the retroflex sounds of Quechua Huanca from articulatory phonetics}

\author{
Richard Anthony Ibarra Valer \\ Universidad Nacional Mayor de San Marcos, Lima, Perú \\ Grupo de Investigación Kawsasun \\ richard.ibarra@unmsm.edu.pe
}

\begin{abstract}
Resumen
El quechua es una lengua muy estudiada por los lingüistas. Se le ha clasificado, principalmente, en distintas variedades de acuerdo a sus características fonémicas y morfémicas. De esta forma, el quechua huanca o wanka es una variedad que presenta, entre otras características, sonidos retroflejos: uno africado y otro fricativo. En consecuencia, estos sonidos, o solo el africado según otros autores, están dentro del inventario fonológico del llamado protoquechua, ya que se trata de sonidos complejos por su articulación.

El objetivo de este artículo es brindar un estado de la cuestión sobre los sonidos retroflejos en el quechua wanka y evaluar las características articulatorias de este tipo de sonidos en otras lenguas andinas como el cauqui y el jaqaru, a la luz de los nuevos avances en el análisis fonético sobre la retroflexión, especialmente, desde el aporte de Hamman (2003) sobre el punto de articulación lingual.
\end{abstract}

Palabras clave: quechua, huanca, sonidos retroflejos, diacronía, fonética articulatoria.

\begin{abstract}
Quechua is a language widely studied by linguists. It has been classified, mainly, in different varieties according to its phonemic and morphemic characteristics. In this way, Quechua huanca or wanka is a variety that presents, among other characteristics, retroflex sounds: one affricate and another fricative. Consequently, these sounds, or only the affricate according to other authors, are within the phonological inventory of the so-called protoquechua, since they are complex sounds due to their articulation.

The objective of this article is to provide a state of the art on retro-reflective sounds in Quechua Wanka and to evaluate the articulatory characteristics of these types of sounds inside of other Andean languages as Cauqui and Jaqaru in light of new advances in phonetic analysis, especially, since the contribution of Hamman (2003) on the point of lingual articulation.
\end{abstract}

Key words: quechua, huanca, retroflex sound, diachrony, articulatory phonetic. 


\section{Richard Anthony Ibarra Valer}

\section{Introducción}

El quechua huanca es una variedad hablada en todo el valle del Mantaro, el cual abarca los distritos de Jauja, Concepción, Huancayo y Chupaca, en el departamento de Junín. Esta variedad pertenece al Quechua I o Wáywash (Torero, 2002) presenta tres subvariedades: el Huaylla-Huanca, el Huaycha-Huanca y el ShaushaHuanca (Cerrón-Palomino, 1976).

Asimismo, los estudios fonéticos que tratan solo estos sonidos retroflejos en esta variedad son escasos y la única evidencia con la que se cuenta es la que fue registrada en trabajos más generales, como gramáticas y manuales de enseñanza. En la mayoría de los estudios precedentes no se ha caracterizado estos sonidos complejos a partir de un análisis fonético exhaustivo, por lo que se trataría de conclusiones basadas más en las impresiones del observador. Sin embargo, esto no sucede por error de los investigadores, sino porque los estudios más detallados recién están cobrando fuerza dentro del campo de la lingüística, pues se proponen mejorar las técnicas para describir o analizar hasta los sonidos más complejos con el auxilio de la tecnología digital.

Un buen ejemplo es el estudio de Carrera Guerrero (2014), quien brinda datos acústicos y articulatorios sobre el sonido fricativo retroflejo sordo de la lengua mazateco. Este autor señala, a partir de palatografías estáticas y lingüogramas, que los sonidos descritos como retroflejos no son articulados con el ápice o subápice de la lengua, sino con los bordes laminales.

Igualmente, Hamann (2003) realiza un estudio sobre los sonidos retroflejos en general y concluye con el cuestionamiento del uso del término «retroflejo» formulando indicadores para reconocer fonética y fonológicamente a los sonidos retroflejos. En ese sentido, tiene en cuenta el modo de articulación, la influencia vocálica y hasta la familia lingüística a la que pertenece una lengua. El autor muestra que la articulación de los sonidos retroflejos no necesariamente implica la flexión hacia atrás de la punta de la lengua.

El objetivo de este artículo es brindar un estado de la cuestión sobre los sonidos retroflejos en el quechua wanka; en consecuencia, se explica cómo estos sonidos son descritos por los autores en base a la fonética articulatoria, no solo en trabajos sincrónicos, sino también diacrónicos. Asimismo, estos datos son relacionados con descripciones sobre otras lenguas andinas que también presentan sonidos retroflejos: el jaqaru y el cauqui para evaluar si existe relación entre los sonidos retroflejos de estas lenguas.

$60 \quad$ Lengua \& Sociedad 
Sincronía y diacronía de los sonidos retroflejos del quechua huanca...

Los sonidos retroflejos son considerados como segmentos complejos, debido a su dificultad y esfuerzo articulatorio. Por lo tanto, estos sonidos, según la variación diacrónica deben de tender a simplificarse debido al principio de economía o del mínimo esfuerzo articulatorio. Este principio se basa, a su vez, en la premisa de que el lenguaje natural debe ser lo más simple posible, pero a la vez lo suficientemente discriminante como para no generar ambigüedad $\mathrm{u}$ «oscuridad» en el mensaje. De modo que, la importancia de este trabajo radica en la problematización que se hace sobre la descripción fonética hecha anteriormente sobre el grupo de sonidos reconocidos como retroflejos, en una lengua andina, específicamente en la variedad huanca del quechua.

En el presente artículo se presenta cuatro apartados. El primero corresponde al marco conceptual, donde se definen la terminología utilizada en el análisis. En el segundo se expone la metodología, en la cual se señala cuál es la muestra elegida y cómo se analizan los datos. En el tercero se presenta el análisis crítico mediante las comparaciones entre los datos obtenidos por varios investigadores sobre los sonidos retroflejos. Y, por último, en el cuarto se expone de manera resumida y concreta las conclusiones, además de algunos alcances pertinentes para futuras investigaciones relacionadas a este tema.

\section{El marco conceptual para el estudio de la retroflexión}

En esta sección expondremos los aportes teóricos de la fonética articulatoria y los conceptos relacionados con una aproximación sincrónica y diacrónica al fenómeno de la retroflexión.

\subsection{La retroflexión desde la fonética articulatoria}

Retroflejo es un término utilizado tradicionalmente para señalar la flexión hacia atrás de la punta de la lengua. (Hamann, 2003). 
Figura 1.

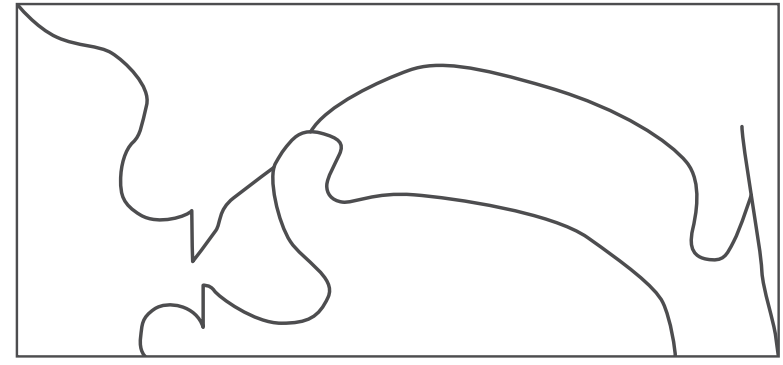

Extraído de Hamann (2003, p. 11)

Sin embargo, el mismo autor citado, señala que en realidad se deben evaluar los sonidos a partir de cuatro indicadores para saber si son retroflejos: apicalidad, posterioridad, cavidad sublingual y retracción. Esta propuesta permite catalogar ciertos sonidos como retroflejos por más que no se flexione la lengua hacia atrás. Por lo cual, Hamann (2003) propone que existen tres tipos de sonidos retroflejos: subapicales, apicales y laminales (o coronales).

Por otro lado, el articulador activo es aquel que presentan movimiento; por ejemplo, el labio inferior o la lengua (distinguiéndose en sus partes). Asimismo, el articulador pasivo es aquel que no presenta movimiento, que es inmóvil, el cual comprende toda la parte superior del tracto vocal desde los labios hasta la faringe.

La lengua se divide en varias partes. En general, la parte más importante es la parte frontal de esta, la cual se denomina corona; además, se divide en dos partes: la punta y la lámina. El ápice o la punta de la lengua comprende la zona casi vertical y la lámina sigue poco después (Ver Fig. 2). Por lo tanto, los sonidos producidos con la punta son llamados apicales y los sonidos producidos con la lámina, laminales o laminares. En consecuencia, se plantea la importancia de reconocer el término punto de articulación lingual para hacer referencia a la parte de la lengua con la que se produce el sonido. Esta propuesta de Hamann (2003) plantea que justamente por esta variación es que se encuentran segmentos que son retroflejos; pero simultáneamente, son distintos por la sección de la lengua con la que se articulan dichos sonidos.

El área debajo de la lengua, enseguida del ápice, es denominada como subápice. Asimismo, existe un espacio debajo de la cuchilla y atrás de los dientes inferiores, al cual se le denomina, por correspondencia, cavidad subapical. 
Sincronía y diacronía de los sonidos retroflejos del quechua huanca...

Después de la lámina, la cual mide aproximadamente de 1 a 1,5 cm (Hamann, 2003), empieza el dorso de la lengua, el cual también es llamado cuerpo de la lengua y se divide en antedorso y posdorso, los cuales prácticamente siempre chocan con el paladar y el velo del paladar, respectivamente, aunque esta distinción no es usada en la mayoría de casos.

Figura 2.

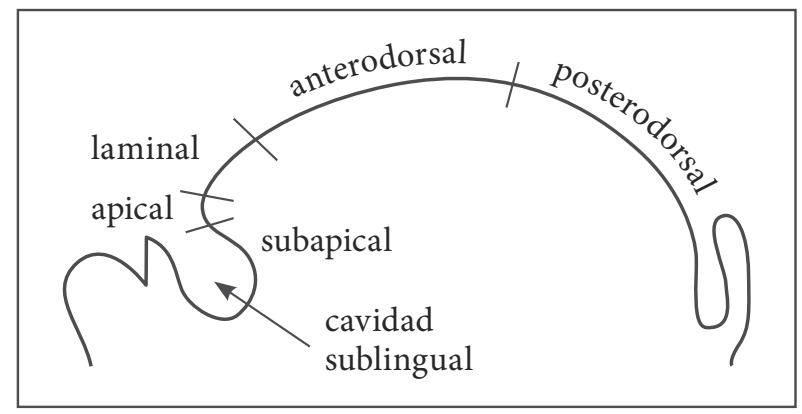

Extraído de Hamman (2003, p. 12).

El alveolo es la cresta o protuberancia que se encuentra inmediatamente detrás de los dientes superiores. Luego de esta, empieza la pendiente del paladar. La zona que se encuentra justo después de dicha cresta es la zona posalveolar. Finalmente, se encuentra el paladar y después el velo del paladar.

Los sonidos producidos en zona del alveolo son llamados alveolares; en la zona posalveolar, posalveolares; en el paladar, palatales, y en el velo del paladar, velares.

Figura 3.

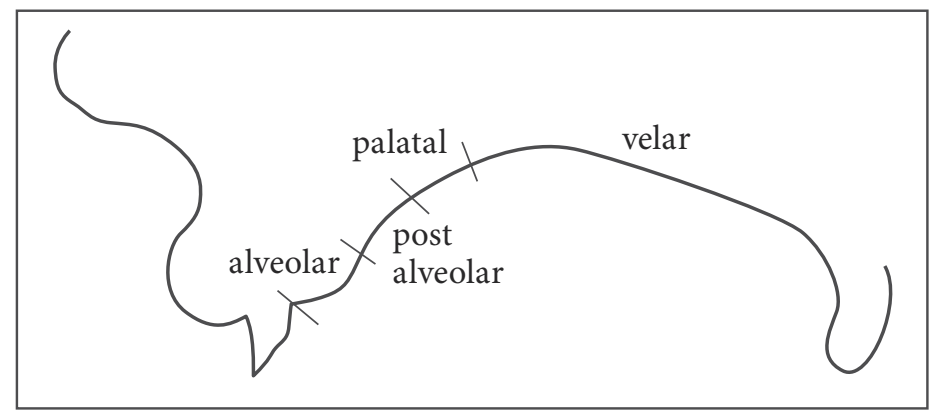

Extraído de Hamman (2003, p. 13). 


\section{Richard Anthony Ibarra Valer}

Este enfoque se expone para interpretar las descripciones y términos utilizados por los investigadores desde un enfoque fonético articulatorio, buscando una correspondencia y resaltando qué tipos de sonidos retroflejos están describiendo los autores.

\subsection{Diacronía, sincronía y el principio de economía articulatoria}

El campo que estudia la variación que se produce por el paso del tiempo a largo plazo es conocida como diacronía; mientras que la sincronía se refiere al campo que estudia el fenómeno lingüístico en su estado durante un solo periodo de tiempo. Por lo tanto, estos términos también se relacionan con el principio del mínimo esfuerzo articulatorio, ya que se entiende que la simplificación de la articulación de un segmento, en este caso retroflejo, en un proceso no marcado; es decir, ocurre de manera constante en muchas lenguas.

Conde (2007) clasifica las investigaciones sobre sociolingüística histórica. Su clasificación será utilizada en este trabajo, pues aporta con la aproximación histórica en una rama de la lingüística que considera que la variación es ingente a nivel micro y depende de muchos factores. Este autor propone dos grandes grupos: 1) estudios en tiempo aparente y 2) estudios en tiempo real. En el primer grupo, se encuentran estudios diacrónicos que intentan reconstruir la evolución de una lengua simulando factores (en este caso, lingüísticos) a partir de trabajos que no tienen el mismo tema de investigación, es decir, otros más generales, y, por otro lado, estudios sincrónicos que observan y describen un fenómeno lingüístico. En el segundo grupo, se encuentran solo estudios diacrónicos que estudian el fenómeno por un largo tiempo para observar la variación o el cambio; estos pueden ser de panel (mismos informantes) o de tendencia (muestra representativa idéntica).

En consecuencia, en este artículo se realiza un estudio diacrónico de tiempo aparente; ya que, el análisis consiste en la interpretación que se hace sobre las descripciones de los sonidos retroflejos que hacen otros autores, los cuales no han trabajado específicamente el tema que se está desarrollando, sino otros más generales. Asimismo, se analizan las descripciones de los sonidos en cuestión que tienen la perspectiva sincrónica, aquellos que son descriptivos, y diacrónica, aquellos que explican la evolución de una lengua o variedad.

Por otro lado, este trabajo toma como fundamento principal para el estudio diacrónico al principio de economía o de mínimo esfuerzo articulatorio. Este se entiende como aquella tendencia o ley natural del propio lenguaje que equilibra

$64 \quad$ Lengua \& Sociedad 
Sincronía y diacronía de los sonidos retroflejos del quechua huanca...

dinámicamente la dificultad de la pronunciación y el uso de un fonema [conservando las oposiciones a nivel perceptual o análisis del habla], según Paredes (2008, p. 167). En suma, Moreno (2002) explica que este principio se ve apoyado por muchos otros factores, como la rapidez del habla, con consecuencias fonéticas articulatorias; aparición de nuevas distinciones sociales, con repercusiones lingüísticas; mantenimiento del estatus quo, por ejemplo, con el que surge una variedad estándar; etc. Este principio opera a nivel sincrónico, con la variación libre - e. g. / $\widehat{\mathrm{t}} / \mathrm{se}$ realiza como [ $\left[\mathrm{t} \int\right]$ o [S] en algunas variedades del castellano-, o diacrónico, con los cambios fonético-fonológicos - e. g. / $/$ / cambió a /x/ en el castellano-.

\section{Metodología}

El presente artículo estudia los sonidos retroflejos desde el paradigma articulatorio a partir de la interpretación de las descripciones que hicieron distintos autores sobre la variedad quechua huanca; además, se apoya en descripciones realizadas sobre los sonidos retroflejos del jaqaru y cauqui. En síntesis, se analiza el sonido africado retroflejo sordo y el sonido fricativo retroflejo sordo desde el punto de vista articulatorio desde el enfoque propuesto por Hamman (2003). Se busca aumentar o dar otro enfoque al conocimiento ya existente sobre los sonidos retroflejos del quechua wanka.

\section{Revisión crítica de los antecedentes sobre la retroflexión en el Huanca}

Los estudios en los que se describen a los sonidos retroflejos que se consideran para el análisis fueron publicados hasta el año 2019. Por tanto, se analiza las descripciones de los sonidos retroflejos del quechua wanka hechas por CerrónPalomino: Gramática quechua Junín-Huanca (1976), El franciscano Raez y la unificación del quechua (1985), Lengua y sociedad en el valle del Mantaro. Primera parte: Quechua fronterizo (1987), Diccionario huanca quechua-castellano castellano-quechua (2018); en el caso del cauqui, se tiene a Oliva: El cauqui: documentación y descripción fonológica de una lengua moribunda (2006); y, por último, en el caso del jaqaru, se tomó en cuenta la investigación de Hardman: Jaqaru: compendio de estructura fonológica y morfológica (1983). 


\section{Richard Anthony Ibarra Valer}

\subsection{Descripción fonético-articulatoria}

A continuación, se presenta los estudios que directamente se orientan a la descripción fonética articulatoria de la retorflexión en tres lenguas andinas; el quechua, el cauqui y el jaqaru.

4.1.1. En el caso del quechua wanka, las primeras descripciones a analizar son las hechas por Cerrón-Palomino (1976). Este autor caracteriza los sonidos retroflejos de manera básica y general; además, explica los procesos fonético-fonológicos que están involucrados con esta peculiar «articulación». En ese sentido, el autor expone lo siguiente:

[...] la presente variedad quechua tiene dos africadas palatales, pero mientras una de ellas es no-retroflexa (es decir /č/, semejante a la ch español), la otra es retroflexa, es decir una consonante cuya articulación requiere de una leve retroflexión del ápice de la lengua (p. 40).

Es decir, entiende que la retroflexión se trata de la producción de un sonido con la parte subapical de la lengua, exactamente con el ápice o punta. Asimismo, explica esta articulación a partir de su parecido con otro, el sonido africado posalveolar, o llamado palatal por dicho autor. Por consiguiente, el quechua wanka presenta oposiciones de estas consonantes por el punto de articulación lingual, además del modo de constricción y el punto de articulación [en el articulador pasivo] —estos dos últimos son utilizados en el Alfabeto Fonético Internacional一.

Además, explica la restricción para el proceso de retroflexivización del sonido africado palatal y, por consiguiente, se entiende la relación entre los sonidos retroflejos y los sonidos palatales (y, por consiguiente, posalveolares), pues este último punto de articulación es el causante de desencadenar algunos procesos fonéticos.

[...] en toda el área que se ha denominado Huaycha-Huanca el fonema /čl, que

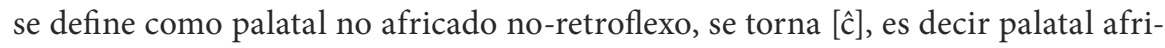
cado retroflexo, cumpliéndose de este modo un proceso de retroflexivización. Este cambio es general y solo se inhibe cuando dicho fonema es seguido por la vocal /i/. [...] Hay que señalar que este cambio tiene otra limitación que consiste en que la / č/ que se encuentre en una misma raíz en la que coaparece una consonante palatal

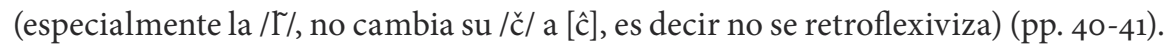

Por último, señala que en las otras dos subvariedades la distinción entre estos dos fonemas se mantiene, pues el proceso de retroflexivización solo se aplica en la subvariedad Huaycha. Este proceso es marcado, pues estos sonidos 
Sincronía y diacronía de los sonidos retroflejos del quechua huanca...

posalveolares tendrían que tender a simplificarse o mantenerse, mas no a complicar su producción.

Como se observa, el autor hace uso de otros símbolos que en la actualidad ya no son utilizados como tal. En el apartado de Equivalencia de signos, CerrónPalomino (1976) explica lo siguiente:

[č] consonante palatal no-retroflexa, como la ch de acechar [asečár].

[ĉ consonante palatal retroflexa, como el grupo $t r$ del español chileno; por ej. en cuatro [kwácô]. [...]

[š] consonante dorso-palatal fricativa, semejante a la sh de Ancash o la sh del inglés shake [šéyk] 'batir'.

[ŝ] consonante alveopalatal fricativa retroflexa, semejante a la $s$ del español peninsular. (p. 27)

En el grupo de segmentos africados se observa que la distinción entre estos sonidos se da a partir del punto de articulación lingual, pues se señala que los dos sonidos son palatales a nivel de punto de articulación.

Por otro lado, en el grupo de los segmentos fricativos se da la distinción no solo a partir del punto de articulación en el articulador pasivo, sino también a partir del punto de articulación lingual. Así, el sonido fricativo [ $\left.\int\right]$ ([̌̌] según el autor) es descrito como «dorso-palatal», lo cual es redundante. A continuación, el sonido [s] ([ŝ] según el autor) es descrito como alveopalatal. De lo último señalado, se infieren las siguientes posibilidades: que el sonido sea alveopalatal o posalveolar y que, por lo tanto, es subapical.

En ese sentido, Cerrón-Palomino (1976) hace la descripción del sonido fricativo de la siguiente manera:

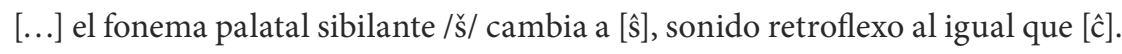
Dicho sonido, cuya articulación se asemeja a la /s/ apical del español peninsular, no aparece sin embargo en un contexto limitado: en contacto con la vocal /i/ cuando forma parte de la misma sílaba y tras la semivocal /y/, aunque esta restricción parece estar relajándose en la localidad de Marco. [...] Esta innovación es producto de un desarrollo interno del huanca y se ha propagado en algunas hablas del quechua de Yauyos (p. 46).

En consecuencia, se entiende que, en la mayoría de los casos, el fonema /// será realizado como [s], excepto cuando esté al lado de los sonidos /i/ y /j/. También, se confirma que la descripción que hace el autor indica que el sonido [s] tiene 


\section{Richard Anthony Ibarra Valer}

como punto de articulación lingual al subápice, pues se hace una comparación con un sonido apical.

Asimismo, Cerrón-Palomino (2018), en el tema de la simbolización de estos sonidos, solo actualiza el usado para el sonido fricativo retroflejo, mas no para los africados, además de utilizar una descripción parecida a la que hizo en 1976.

[č ] Simboliza a la africada palatal, como la <ch > castellana. Así, en la voz /chusu/ 'pequeño'.

[î] Representa a la africada palatal retrofleja, parecida al sonido que adquiere el grupo consonántico $<$ tr $>$ en algunos dialectos del castellano, como el chileno, donde tren se pronuncia [ĉen]. Ocurre, por ejemplo, en la palabra [î̀icu] 'preñada'.

[s] Simboliza a la sibilante apical ligeramente retrofleja, típica del quechua-huanca, como en [sansa] 'ascua'.

[š Representa a la sibilante palatal sorda, como el dígrafo $<$ sh $>$ del topónimo quechua Áncash; en el huanca la tenemos, por ejemplo, en [šimi] 'boca'.

(2018, p. 10)

Como se observa, se eliminan algunos términos redundantes y se deja en claro que el sonido fricativo retroflejo [s] es apical; por lo tanto, ahora se describe como un sonido apical o subapical retroflejo, es decir, no se precisa cuál es el punto de articulación lingual.

4.1,2. En el caso de la lengua cauqui, se tiene el trabajo elaborado por Oliva León (2006). En esta tesis, se documenta esta lengua y se discute los postulados de investigadores anteriores. El cauqui presenta el sonido africado retroflejo y, en consecuencia, como es característico de las lenguas aru, también en sus formas complejas, aspirada y glotalizada. Entonces, el cauqui tiene tres sonidos retroflejos. Además, presenta oposición con el sonido africado alveopalatal y el sonido africado posalveolar (palatal según el autor). Cabe resaltar que esta lengua se habla en Cachuy, Canchán y Chavín, en el distrito de Tupe, provincia de Yauyos, Lima.

Oliva (2006) utiliza los rasgos postulados desde la lingüística generativa para poder describir los sonidos del cauqui. Por ende, se tiene la siguiente adaptación:

Tabla 1. Sonidos del cauqui

\begin{tabular}{l|l|l|l|l}
\hline Rasgos & tç & $\check{\mathbf{c}}$ & $\hat{\mathbf{c}}$ & $\check{\mathbf{s}}$ \\
\hline Continuo & - & - & - & + \\
\hline Realización retardada & + & + & + & \\
\hline
\end{tabular}


Sincronía y diacronía de los sonidos retroflejos del quechua huanca...

\begin{tabular}{l|l|l|l|l}
\hline Rasgos & tç & č & $\hat{\mathbf{c}}$ & s \\
\hline Labial & - & - & - & - \\
\hline Anterior & + & - & - & - \\
\hline Posterior & & - & - & \\
\hline Coronal & + & + & + & + \\
\hline Distribuido & + & + & - & + \\
\hline Glotis dilatada & - & - & - & - \\
\hline Glotis constreñida & - & - & - & - \\
\hline Alto & & + & + & \\
\hline
\end{tabular}

Adaptación de Oliva (2006, p. 24).

Además, define a los sonidos en cuestión de la siguiente forma: $/ \mathfrak{t} c ̧ /$ como africada alveopalatal simple, /č/ como africada palatal plana simple, /ĉ/ como africada palatal retrofleja simple y /š/ como fricativa palatal. Esta oposición es fundamentada a partir de los rasgos desde la corriente generativa, específicamente del modelo de geometría de rasgos o fonología autosegmental. Estos rasgos tienen jerarquía, además de una correspondencia directamente articulatoria.

Por lo tanto, se observa que: a) la diferencia entre los sonidos africados tiene que ver con los rasgos [anterior] y [distribuido]; b) además del uso de [continuo], el segmento fricativo no presenta algunos rasgos que su par africado sí, [posterior] y [alto], lo cual no permite inferir una razón evidente — pues [realización retardada] da a entender que existen dos momentos, mientras que en los otros dos casos no existe una explicación implícita o evidente sobre la ausencia de valores en estos rasgos- . Por lo tanto, se describe al sonido africado retroflejo como coronal, así que se podría hipotetizar que el punto de articulación lingual es apical, pues no es un distribuido y a la vez es coronal, si en caso sería subapical, el sonido en cuestión no podría ser coronal; además, es un sonido posalveolar, pues es alto, generando así una contradicción, pues se espera que el sonido sea subapical, mas no coronal. Aunque esto último podría deberse al modelo autosegmental que utiliza el autor, pues, dentro del modelo autosegmental, no existe el rasgo [retroflejo].

Del mismo modo, Oliva (2006) indica que, ocasionalmente, los segmentos retroflejos alternan libremente con los sonidos africados dentoalveolares, los cuales están presentes en el jaqaru. Este caso se podría deber a que este sonido estaba sufriendo el cambio $\widehat{\mathrm{t}} \int>\hat{\mathrm{f}} \mathrm{s}$, pero este no se concretó en el cauqui, mas 
sí se concluyó en el jaqaru. Además, afirma que el sonido africado retroflejo no presenta alófonos producto de un proceso fonético, es decir por la influencia de algún otro segmento, sino que siempre es el mismo en todos los contextos posibles. Sin embargo, en los ejemplos que muestra para la forma simple, no se presenta ninguna entrada en la que el sonido retroflejo está adyacente a la vocal/i/ o algún elemento palatal, como lo es / $\mathrm{j} /$ (/y/ según la simbología utilizada por el autor) $\mathrm{o} / \mathrm{K} / \mathrm{y}$, por el contrario, se presenta ejemplos de la vocal /i/ con los sonidos africados retroflejos complejos, tanto aspirado como glotalizado. En suma, en dicho trabajo, Oliva (2006) no presenta procesos fonéticos que afecten a las vocales por la presencia de algún sonido retroflejo, lo cual no sería posible, pues existe una imposibilidad articulatoria entre los sonidos retroflejos y la vocal /i/ (o algún otro segmento parecido que implique palatalidad). Hamann (2003) explica que, por implicancias articulatorias, cuando un sonido retroflejo se encuentra con la vocal /i/, alguno de los sonidos tiene que cambiar: o bien el segmento retroflejo se simplifica, generando un sonido palatal o posalveolar; o bien la vocal desciende.

4.1.3. En el caso del jaqaru, Hardman (1983) trabaja la fonología y morfología de esta lengua. En este estudio, la autora señala que en esta lengua existe un segmento palatal retroflejo, el cual es considerado como oclusivo/africado y, al igual que el cauqui, presenta su forma aspirada y glotalizada.

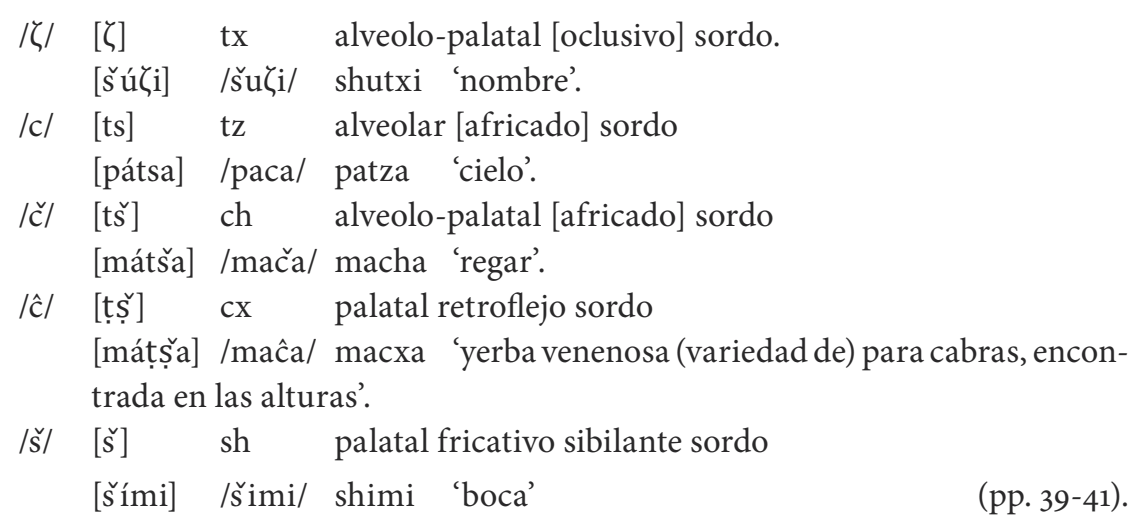

Se observa que el primer sonido de la cita anterior es un sonido oclusivo posalveolar o palatalizado, es decir $\left[\mathrm{t}^{\mathrm{j}}\right]$. Por otro lado, la autora indica que el sonido retroflejo tiene como punto de articulación a la zona del paladar, por lo cual se puede inferir que es la zona posalveolar específicamente y por ello la producción de este sonido se realiza con el subápice de la lengua, mas no con el ápice. 
Sincronía y diacronía de los sonidos retroflejos del quechua huanca...

Hardman (1983) no presenta alófonos de este sonido retroflejo, lo cual se contrapone a lo que plantea Oliva (2006). Este último autor propone que en el jaqaru los sonidos retroflejos sufren un proceso y como resultado pasan a ser dentoalveolares. Esta información ayuda a configurar el cambio que han estado sufriendo estos sonidos, en el cual se están simplificando, pues la lengua se está adelantando con respecto al punto de articulación.

Con respecto a los procesos fonéticos, Hardman (1983, p. 50) sostiene que el grupo de sonidos retroflejos «cierran» las vocales, pues, según la autora, estos segmentos determinan bandas de frecuencias o formantes difusas que se corresponden con tensión en las vocales, ya que articulatoriamente no las relaja ni las redondea.

Asimismo, Hardman (1983) describe acústicamente estos sonidos usando los rasgos acústicos de Jackobson, pero desde la perspectiva no binaria, pues se establece oposición a partir de una escala acústica, la cual, en consecuencia, tiene implicancias articulatorias.

Tabla 2. Tabla de rasgos acústicos de algunos sonidos del jacaru

\begin{tabular}{l|l|l|l|l}
\hline & grave & no-grave & no-agudo & agudo \\
\hline$\zeta$ & $\mathrm{O}$ & $\mathrm{O}$ & - & + \\
\hline $\mathrm{c}$ & $\mathrm{O}$ & $\mathrm{O}$ & + & - \\
\hline$\check{c}$ & $\mathrm{O}$ & $\mathrm{O}$ & - & + \\
\hline$\hat{\mathrm{c}}$ & $\mathrm{O}$ & - & - & $\mathrm{O}$ \\
\hline$\check{s}$ & $\mathrm{O}$ & $\mathrm{O}$ & - & + \\
\hline
\end{tabular}

Adaptado de Hardman (1983, p. 53).

De ahí que Hardman (1983) propone una escala de siete grados: G+, G, G-, N, A-, A y A+. Esta escala se basa en rasgos acústicos (Ver Cuadro 1) y agrupa a los sonidos de acuerdo a la intensidad que presenten: Muy grave, grave, poco grave, neutro, poco agudo, agudo y muy agudo, respectivamente con la simbología utilizada por la autora.

Por eso, el grupo de sonidos retroflejos, según la investigadora, se encuentran en G-, esto debido a la resonancia que se produce en la cavidad subapical por el punto de articulación lingual. En oposición, los sonidos posalveolares (o palatales) del inventario fonológico del jaqaru se encuentran en la escala A o A+. 


\section{Richard Anthony Ibarra Valer}

La correspondencia entre la data articulatoria previamente explicada y la data acústica se corresponde. Si bien se dice que el sonido retroflejo es apical subapical, esto no significa que estos sonidos retroflejos tengan que ser $\mathrm{A}$ o A+, pues aquí interviene la cavidad sublingual, la cual funciona como otra caja de resonancia dentro de la cavidad oral, dividida por la lengua. Además, por la misma cuestión articulatoria explicada, estos segmentos retroflejos no podrían llegar a ser $\mathrm{G}+\mathrm{o}$ $\mathrm{G}$, pues no son sonidos posteriores o dorsales.

\subsection{Recuento histórico: esbozo de la aparición de los sonidos retroflejos en el quechua wanka}

La evidencia muestra que existen sonidos retroflejos en lenguas o variedades que se hablan en la zona central del país. Entonces, ¿estos sonidos surgen por préstamo (influencia externa) o innovación (cambio interno) en el quechua huanca? Esta parte del análisis da algunos alcances sobre este tema.

El quechua es una lengua que ha estado y está en constante contacto con muchas otras y, en consecuencia, ha ido cambiando mucho a través del tiempo, de acuerdo a las sociedades que ocuparon el mismo o territorio cercano, tales como Tiahuanaco, Huari, Culli, etc. En suma, los sonidos retroflejos implican una articulación costosa y, normalmente, las lenguas tienden a simplificarlos, es decir, a hacer menos costosos y más simples de articular. En el quechua huanca, estos sonidos se preservaron hasta la actualidad, pues existe evidencia de que ya existían desde hace mucho, según los registros que dejó el franciscano Raez (Cerrón-Palomino, 1985).

Primero, Torero (2002, p. 58) propone que el sonido africado retroflejo está en el inventario fonológico del protoquechua. En consecuencia, se asume que el quechua wanka es una variedad conservadora.

Por otro lado, Cerrón-Palomino (1976) afirma que el sonido fricativo retroflejo surge dentro de la misma variedad y que esta influye a otras variedades, como es el quechua de la sierra de Lima, Yauyos.

\footnotetext{
Esta innovación es producto de un desarrollo interno del huanca y se ha propagado en algunas hablas del quechua de Yauyos. (p. 46)
}

Además, esta oposición entre retroflejo y no retroflejo no es homogéneo, pues en una de las tres subvariedades se prefiere la complejidad de la articulación y la distinción a partir del contexto de los pares mínimos existentes.

$72 \quad$ Lengua \& Sociedad 
Sincronía y diacronía de los sonidos retroflejos del quechua huanca...

En suma, la oposición palatal no retroflexa versus palatal retroflexa se elimina en el Huaycha-Huanca, al par que en el Huaylla y en el Shausha la distinción se mantiene de manera consistente (p. 41).

Cerrón-Palomino (1976) plantea que el fonema / // se retroflexiviza en todos los contextos, excepto adyacente con /i/ y otros sonidos palatales. Es decir, el sonido retroflejo fricativo está en el plano fonético, mas no fonológico. Esta idea no es compartida por todos los investigadores de quechua wanka. Rick Floyd (1997) plantea que el sonido fricativo retroflejo /s/ es un fonema, al igual que / $/$. Sea cualquiera de los dos casos, Hamann (2003) plantea que debe de existir oposiciones entre las categorías lingüísticas que maneja la lengua para que los sonidos sean funcionales, no solo a partir de pares mínimos, sino también a partir de la relación articulatoria que hay entre estos. Es decir, es posible la existencia de sonidos fricativos retroflejos, ya que existe oposición con el grupo no retroflejo dentro del grupo de sonidos fricativos; además de que existe esta misma oposición entre los sonidos africados.

Así mismo, Cerrón-Palomino (1987) reconoce que para el protohuanca existe el sonido africado retroflejo a nivel fonológico. Por otro lado, no reconoce al sonido fricativo posaveolar como parte de este inventario, pues sostiene que la oposición de retroflexión entre los sonidos fricativos se daba con el segmento fricativo alveolar ${ }^{\star} / \mathrm{s} /$.

Como quiera que para el PQ se han postulado las sibilantes $* / s / y * / s /$, donde la última se corresponde con nuestra ${ }^{\star} / \hat{s} /$, ello implicaría que ésta sufrió un cambio a partir de la palatal. Creemos, sin embargo, que las cosas sucedieron al revés. Nuestra creencia se basa en por lo menos tres retazos de evidencia de naturaleza tanto teórica, como dialectal y filológica (p. 7).

Esta oposición da cuenta de que esta retroflexión, internamente en la lengua, se distingue en las dos formas en la que aparece. Es decir, la oposición entre sonidos africados se da a partir del carácter palatal, posalveolar específicamente, de estos sonidos; por lo tanto, se estaría hablando de un sonido africado retroflejo subapical. Por otro lado, en los sonidos fricativos, si solo hay oposición con el sonido alveolar, implica que la retroflexión se da a nivel coronal; es decir, el segmento fricativo retroflejo es apical. Sin embargo, ya sincrónicamente, si también existe $/ \int /$, la oposición es distinta, pues el segmento fricativo retroflejo tiene que ser laminal. De esta última hipótesis, se entiende que el sonido fricativo retroflejo no podría ser subapical, pues existe oposición con dos sonidos, uno alveolar y otro 


\section{Richard Anthony Ibarra Valer}

posalveolar, los cuales implican una articulación coronal y palatal (posteriorizada). Esta última hipótesis muestra la posibilidad que el quechua huanca podría tener dos sonidos retroflejos que son distintos en el punto de articulación lingual.

Volviendo a los pueblos del Valle, debe señalarse que la nueva demarcación introducida por los incas significa el desmembramiento político-administrativo del territorio étnico de los huancas, mas no del de los shaushas, que permanece inafectado. Como veremos, esta segunda escisión dará como resultado una nueva fragmentación lingüística.

En efecto, es dentro de la parcialidad de Lulin-Huanca, y sólo en ella, que se producen dos cambios: el de ${ }^{\star} / \check{c} />\hat{c}$ y el de /l/ > č (cf. 2.31.3 y 2.31.4). Encontramos aquí, como se ve, una nueva correlación lingüístico-administrativa inobjetable. Ahora bien, mientras que para la primera mutación no tenemos más remedio que aceptar una motivación interna, para la segunda (que, conforme se vio, pareciera igualmente haberse originado por presión del sistema) hay, quizás, razones de naturaleza externa (1987, p. 27).

La retroflexivización del protofonema africado posalveolar sordo es asumido por Cerrón (1987) como un proceso interno. Este proceso también es replicado para el segmento fricativo posalveolar. Sin embargo, la motivación interna planteada por este autor es insuficiente, pues si fuera así, si solo es un proceso lingüístico, este tendría que tender a simplificar de acuerdo al criterio de economía articulatoria o naturalidad. Por ende, la evidencia hasta ahora indica que estos sonidos ya estaban presentes en el protoquechua, al menos el segmento africado; pero su preservación tiene que haberse debido a otros factores lingüísticos como el contacto con otras lenguas o variedades de quechua, y/o sociales, dentro de la misma comunidad huanca o por imposición externa.

De hecho, las variedades quechua que conservan el sonido africado retroflejo, según Torero $(1964,16)$, son las habladas en Yauyos, Canta, y parte de Chancay y Cajatambo en Lima; Pasco; San Rafael, provincia de Ambo en Huánuco; Sihuas y Corongo en Áncash; provincia de Cajamarca, y en la serranía de Ferreñafe en Lambayeque. Asimismo, se debe considerar que las lenguas en contacto pueden influir en la conservación de los sonidos retroflejos o en la retroflexivización de otros segmentos. Por lo tanto, se tiene al jaqaru que presenta segmentos retroflejos, sobre los cuales Hardman (1983) no postula nada y, en el caso del cauqui, Oliva (2006) señala que esta lengua es más innovadora que el jaqaru, como se observa en la siguiente cita:

$74 \quad$ Lengua \& Sociedad 
Sincronía y diacronía de los sonidos retroflejos del quechua huanca...

Siguiendo el razonamiento de Cerrón-Palomino (2000: 124-128), que explica la evolución de la forma alveolar a la palatal, dicha correlación corrobora el cambio /t/ > / fç/, donde el cauqui tiene una conducta más innovadora que el jacaru (p. 97).

Sin embargo, sería apresurado hipotetizar que el quechua wanka haya prestado el sonido africado retroflejo a estas dos lenguas, sobre todo por el trato distinto que reciben estos sonidos en cada lengua, pues en la familia aru se tiende a establecer oposición consonántica con sonidos simples, glotalizados y aspirados, lo cual se cumple en el cauqui y el jaqaru con el segmento retroflejo; pero, lo problemático, es que no existen sonidos retroflejos en el aymara, el cual también pertenece a la familia aru. Aunque esto último es concordante con el repertorio fonológico del quechua sureño, el cual tampoco presenta sonidos retroflejos.

Finalmente, se sabe de algunos grupos de personas retiradas de su lugar de origen por la fuerza, los llamados mitmas, en la época del imperio incaico que fueron movidas a territorio wanka; por ejemplo, según Cerrón (1987), los lupacas, quienes eran un pueblo aymara y, por consiguiente, hablaban una lengua aru (familia a la que también pertenece el cauqui, el jaqaru y el aymara); los cashamalcas, desde el norte de lo que actualmente es Perú, además tenían como lengua al culli; los chachas, de las tierras altas de lo que actualmente es el departamento de Amazonas; los cañaris, que venían desde el norte, del actual Ecuador; los llaguas (o yaguas), los cuales venían de las riberas del río Amazonas. No se sabe hasta qué punto estas otras lenguas y variedades quechua, la cual era una lengua necesaria para el intercambio comercial en la época, influyeron en el quechua huanca, ya que no existe documentación lingüística de muchas de las lenguas mencionadas anteriormente y tampoco hay mucha evidencia para saber cómo fueron o cuál era su inventario fonético-fonológico, si tenían sonidos retroflejos o parecidos.

Por último, sobre el segmento fricativo retroflejo, Cerrón-Palomino (1987: 7) señala que existe registro sobre este sonido en algunos distritos de Yauyos y que en Cajamarca también aparece un sonido parecido en variación libre, además de mucha más información filológica. Por tanto, se asume que este sonido es antiguo dentro del repertorio fonético-fonológico del quechua huanca.

En la actualidad, el jaqaru y el cauqui siguen siendo estudiados en muchas ramas, aparte de la lingüística. Las investigaciones de Lovón (2009), en el campo de la lingüística; Rehecho et al. (2011), en el campo de la farmacología, y CruzRíos (2019), en el campo de la biología, sobre el jaqaru son muy importantes para la misma comunidad que habla esta lengua y para la comunidad científica. Del 


\section{Richard Anthony Ibarra Valer}

mismo modo, el trabajo de Oliva (2006) sobre el cauqui es uno de los más recientes e importantes que sirven como documentación de dicha lengua.

Del mismo modo, el quechua huanca es una variedad que necesita ser mucho más estudiada; ya que, dentro de la tradición investigativa de la Lingüística Quechua o Andina, hay muy pocos estudios sobre este dialecto, por lo mismo que es una variedad en peligro de extinción. Los trabajos más recientes sobre esta variedad son los de Cerrón-Palomino (2018) y Pearce y Heggarty (2011), también trabajos como los de Adauto (2018), quien trabaja un tema relacionado a la misma comunidad huanca, mas no sobre su lengua, y comparte información sociolingüística de manera indirecta.

En general, estos últimos trabajos sirven de referencia para encontrar data sobre los sonidos retroflejos a partir de la lengua en uso; en consecuencia, no solo se propicia la investigación sobre la fonética y fonología de estas lenguas, la cual es escasa, sino que también en otros muchos campos de la lingüística.

\section{Algunas conclusiones}

A partir de las descripciones brindadas por los autores, se puede concluir que el quechua huanca tiene un sonido africado retroflejo subapical, el cual tiene como base a la oposición palatal que existe entre los sonidos africados. Del mismo modo, para los sonidos fricativos, se manejan dos posibilidades: el primero, que el sonido fricativo retroflejo sea apical, debido a la implicancia articulatoria coronal; o, en todo caso, que el sonido fricativo retroflejo sea laminal, pues hay oposición entre tres sonidos, debido a la implicancia articulatoria coronal y palatal de estos. Asimismo, las otras descripciones de los segmentos retroflejos dan como resultado que, en el jaqaru, este sonido es subapical, mientras que, en el cauqui, este sonido es apical.

De la evaluación presentada, se concluye que la hipótesis más aceptada por la comunidad científica sobre el origen de los segmentos retroflejos del quechua huanca es que surgieron de manera interna, ya que esta variedad es conservadora (por el sonido africado) y a la vez innovadora (por el sonido fricativo). Al parecer, de manera similar, tanto en el cauqui como en el jaqaru también surgieron internamente; por lo tanto, toda esta zona de la sierra central del país contaría con lenguas que tienen sonidos retroflejos. Este hecho podría ser un indicio de que alguna de estas lenguas haya tenido que influir en otras y no que surgieron por innovación como parte de un proceso interno. 
Sincronía y diacronía de los sonidos retroflejos del quechua huanca...

Sin embargo, faltan estudios fonéticos articulatorio-acústicos que muestren, por ejemplo, que los sonidos retroflejos del quechua huanca son los mismos que los de otras variedades quechuas o, es más, en otras lenguas originarias, no solo del Perú (como el jaqaru, cauqui, etc.), sino también de otras zonas de esta parte del continente, como el mapudungún (mapuche) o el chipaya desde el punto de articulación lingual.

Además, tampoco se cuenta con documentación de las lenguas de los pueblos que fueron mitmas o fueron cercanos al valle del Mantaro en la época incaica y preincaica, como el guancavilca o el culli y otros. Por ello, la realización de trabajos en lingüística histórica sobre estas lenguas, así como sobre las mencionadas en el párrafo anterior servirían para fortalecer o debilitar alguna de las hipótesis sobre el origen de los sonidos retroflejos y, en general, sobre otras conjeturas propuestas en la lingüística histórica quechua y andina.

\section{Referencias bibliográficas}

Adauto Rojas, J. F. (2018). Acompañamiento pedagógico en las áreas de matemática y comunicación de la Institución Educativa Pública Túpac Amaru de Huancayo (Trabajo académico para optar por el Título Profesional de Segunda Especialidad en Gestión Escolar con Liderazgo Pedagógico). Lima: UsIL.

Carrera Guerrero, H. (2014). Retroflexión y palatalización en el Mazateco de San Lorenzo Cuaunecuiltitla, Oaxaca. Workshop on the Sound Systems of México and Central America. México.

Cerrón-Palomino, R., y Sihuay, J. C. (1973). Lexicón wanka-español (N. $\left.{ }^{\circ} 10\right)$. Lima: Centro Investigación de Lingüística Aplicada.

Cerrón-Palomino, R. (1975). Hispanismo en el quechua Wanka. Lima: Centro de Investigación de Lingüística Aplicada.

Cerrón-Palomino, R. (1976). Gramática quechua Junín-Huanca. Lima: Ministerio de Educación.

Cerrón-Palomino, R. (1985). El franciscano Raez y la unificación del quechua. Anthropologica del Departamento de Ciencias Sociales, 3(3), 203-246.

Cerrón-Palomino, R. (1987). Lengua y sociedad en el valle del Mantaro. Primera parte: Quechua fronterizo. Amerindia, 12, 33-93.

Cerrón-Palomino, R. (2016). Más allá de la función distintiva: la palatalidad con valor expresivo en el quechua. Indiana, 33(1), 27-37. 


\section{Richard Anthony Ibarra Valer}

Cerrón-Palomino, R. (2018). Diccionario huanca quechua-castellano castellano-quechua. Lima: Instituto Riva-Agüero.

Chacón Sihuay, J. (1973). Quechua wanka (transcripción fonética, fonémica y grafémica de textos) (Vol. 1 y 2). Lima: Centro de Investigación de Lingüística Aplicada.

Chacón Sihuay, J. (1979). Lexicón huanca-español (complemento). Lima: Centro de Investigación de Lingüística Aplicada.

Conde Silvestre, J. C. (2007). Sociolingüística histórica. España: Gredos.

Cruz-Ríos, I. V. (2019). Conocimiento local e importancia del uso de la flora por la comunidad Jaqaru, distrito de Tupe, Lima (Tesis de lincenciatura). Universidad Nacional Mayor de San Marcos, Lima.

Floyd, R. (1997). La estructura categorial de los evidenciales en el quechua wanka. Lima: ILV Perú.

Hamann, S. R. (2003). The phonetics and phonology of retroflexes [Disertación doctoroal].

Hamann, S. (2004). Retroflex fricatives in Slavic languages. Journal of the International Phonetic Association, 34(1), 53-67.

Hardman, M. J. (1983). Jaqaru: compendio de estructura fonológica y morfológica. Lima: IEP Ediciones.

Hussain, Q., Proctor, M., Harvey, M., y Demuth, K. (2017). Acoustic characteristics of Punjabi retroflex and dental stops. The Journal of the Acoustical Society of America, 141(6), 4522-4542.

Lovón Cueva, M. A. (2009). Hacia una teoría de la complejidad: estudio etnolingüístico y cognitivo de la correlación entre los platos típicos tupinos y su construcción lexical en la lengua jaqaru (Tesis de licenciatura). Universidad Nacional Mayor de San Marcos, Lima.

Moreno Cabrera, J. C. (2002). El motor de la economía lingüística: de la ley del mínimo esfuerzo al principio de la automatización retroactiva. Revista espanola de lingüística, 32(1), 1-32.

Oliva León, D. (2006). El cauqui: documentación y descripción fonológica de una lengua moribunda (Tesis de licenciatura). Pontificia Universidad Católica del Perú.

Paredes Duarte, J. (2008). El principio de economía lingüística. Pragmalingüística, $15,7-27,166-178$. 
Sincronía y diacronía de los sonidos retroflejos del quechua huanca...

Pearce, A. J., y Heggarty, P. (2011). «Mining the Data» on the HuancayoHuancavelica Quechua Frontier. In History and Language in the Andes (pp. 87-109). New York: Palgrave Macmillan.

Rehecho, S., Uriarte-Pueyo, I., Calvo, J., Vivas, L. A., y Calvo, M. I. (2011). Ethnopharmacological survey of medicinal plants in Nor-Yauyos, a part of the Landscape Reserve Nor-Yauyos-Cochas, Peru. Journal of ethnopharmacology, 133(1), 75-85.

Torero, A. (1964). Los dialectos quechuas. Lima: Universidad Nacional Agraria La Molina.

Torero, A. (2002). Idiomas de los Andes: lingüística e historia (N. 162$)$. Lima: Instituto Francés de Estudios Andinos. 